\title{
Construction et évaluation d'un programme d'éducation thérapeutique du patient dans le domaine de la douleur liée au cancer
}

\section{Elaboration and evaluation of a therapeutic education program in cancer pain management}

\section{Synthèse}

Virginie Prevost ${ }^{1,2}$, Bénédicte Clarisse ${ }^{2}$, Natacha Heutte ${ }^{2,3}$, Alexandra Leconte ${ }^{2}$, Cécile Bisson $^{4,5}$, Rachel Bignon ${ }^{5,6}$, Sonia Cauchinn ${ }^{5,7}$, Maryline Feuillet ${ }^{5,8}$, Sylvie Gehanne ${ }^{5,8}$, Maud Gicquère ${ }^{2,5}$, Marie-Christine Grach ${ }^{2,5}$, Cyril Guillaumé, ${ }^{5,9}$, Christine Le Gal ${ }^{5,10}$, Joelle Le Garrec $^{5,7}$, Franck Lecaer ${ }^{5,11}$, Isabelle Lepleux ${ }^{5,12}$, Anne-Laure Millet ${ }^{5,11}$, Marie-Claude Ropartz $^{5,13}$, Nathalie Roux ${ }^{5,9}$, Virith Sep Hieng ${ }^{5,6}$, Carole Van Delook ${ }^{5,10}$, Constance Bechet ${ }^{14}$, Aline Le Chevalier ${ }^{5,13}$, Claire Delorme ${ }^{4,5}$

${ }^{1}$ UMR 1086 INSERM, Unité de Recherche Interdisciplinaire pour la Prévention et le Traitement des Cancers «ANTICIPE » et Université de Caen Normandie, Caen, France

${ }^{2}$ Centre Régional de Lutte Contre le Cancer François Baclesse, Caen, France

${ }^{3}$ Normandie Univ, UNIROUEN, CETAPS EA 3832, Mont Saint Aignan, France

${ }^{4}$ Centre Hospitalier, Bayeux, France

${ }^{5}$ Réseau Régional Douleur en Basse-Normandie, Bayeux, France

${ }^{6}$ Centre Hospitalier, Lisieux, France

${ }^{7}$ Centre Hospitalier Intercommunal Alençon-Mamers, Alençon, France

${ }^{8}$ Centre Hospitalier, Saint-Lô, France

${ }^{9}$ Centre Hospitalier Universitaire, Caen, France

${ }^{10}$ Centre Hospitalier, Argentan, France

${ }^{11}$ Centre Hospitalier, Flers, France

${ }^{12}$ Centre Hospitalier, Cherbourg, France

${ }^{13}$ Centre Hospitalier, Avranches-Granville, France

${ }^{14}$ Pharmacie Emile Zola, Paris, France 
Correspondance : Virginie Prevost, UMR 1086 INSERM Unité de Recherche Interdisciplinaire pour la Prévention et le Traitement des Cancers « ANTICIPE », Centre Francois Baclesse, Av. du Général Harris, 14076 CAEN cedex 05 ; e-mail : virginie.prevost@unicaen.fr ; tel : +33.231.45.52.15 


\section{RESUME}

La douleur est l'un des symptômes les plus redoutés par les patients atteints de cancer. Elle reste insuffisamment soulagée et retentit sur leur qualité de vie. L'éducation thérapeutique du patient (ETP) constitue une approche pertinente pour répondre à cette problématique en permettant au patient de développer des compétences pour mieux gérer sa douleur. En BasseNormandie, la prise en charge de la douleur liée au cancer s'appuie notamment sur deux réseaux structurés permettant ainsi la proximité et l'accessibilité pour tous. Dans ce contexte, nous avons construit un large programme de recherche s'inscrivant dans une politique régionale de santé, en cinq étapes : (1) Formation à l'ETP de 10 binômes médecins/infirmières ; (2) Identification des attentes éducatives des patients et des proches dans le domaine de la douleur ; (3) Conception et optimisation d'un programme ETP ; (4) Etude Pilote Régionale permettant d'évaluer la faisabilité, la qualité et la transférabilité du programme ; (5) Evaluation du programme d'ETP par randomisation comparative interventionnelle menée à l'échelle nationale. L'objectif de cet article est de présenter la stratégie de notre approche et plus particulièrement les objectifs, la méthodologie et les résultats attendus des phases de recherche (étapes 2,4,5). A l'issue des cinq étapes, notre ambition est de démontrer l'efficacité du programme éducatif pour améliorer les compétences des patients leur permettant de mieux gérer leur douleur. La finalité à long terme est de diffuser la démarche éducative avec une modification des pratiques apportant un bénéfice réciproque pour les soignants et les patients. 


\begin{abstract}
Pain, one of the most feared symptoms for patients with cancer, remains insufficiently alleviated and impairs quality of life. Therapeutic patient education (TPE) is a relevant approach to this problem while allowing patients to develop skills to better manage their pain. In the "Basse-Normandie" French region, the management of pain relies on two organized networks, thus allowing proximity and accessibility for all concerned. In this context, our team has begun a broad five-step research program that is part of a regional health policy: (1) Training in TPE of 10 doctor / nurse pairs; (2) Identification of educational expectations of patients and their relatives in the field of cancer pain; (3) Design and optimization of a TPE program dedicated to cancer pain ; (4) Regional pilot study aiming to assess the feasibility, quality and transferability of the program; (5) Evaluation of the TPE program by interventional comparative randomization at the national level. This article aims to present the program which originality and strengths are based on collaborative work between health stakeholders. Objectives, methodology and expected results of the research phase (stages 2,4,5) are notably developed. The main expected outcomes are to prove the effectiveness of the program in improving the knowledge and skills of patients in the field of pain cancer in order to promote their adherence to treatment and, consequently, to enable them to better manage it. The long-term objective is to disseminate the educational approach by modifying practices that provide a mutual benefit for caregivers and patients.
\end{abstract}

Mots clés : Education thérapeutique du patient; Douleur liée au cancer; Gestion de la douleur; Evaluation de la douleur

Keywords : Therapeutic patient education; Cancer pain; Pain management; Pain assessment 


\section{DEFINITION ET ORGANISATION DE LA DEMARCHE EDUCATIVE}

Selon la définition de l'Organisation Mondiale de la Santé [1] revue par la Haute Autorité de Santé (HAS) : «L'éducation thérapeutique du patient (ETP) vise à aider les patients à acquérir ou maintenir les compétences dont ils ont besoin pour gérer au mieux leur vie avec une maladie chronique... Ceci a pour but de les aider (ainsi que leurs familles) à comprendre leur maladie et leur traitement, collaborer ensemble et assumer leurs responsabilités dans leur propre prise en charge, dans le but de les aider à maintenir et améliorer leur qualité de vie » [2]. Elle est légalisée en France dans le cadre de son inscription dans l'article 84 de la loi du 21 juillet 2009 portant réforme de l'Hôpital et relative aux Patients, à la Santé et aux Territoires (HPST) qui en précise les principes et les modalités [3].

La démarche éducative dépasse très largement le domaine de l'information mise à disposition du patient et son accompagnement. Elle se caractérise notamment par la notion d'appropriation de compétences par le patient et de l'évaluation de ces compétences. L'objectif est donc, dans un contexte basé sur une relation d'échange, de confiance et de partenariat entre le soignant éducateur et le participant au programme éducatif, de rendre le patient expert de sa maladie. La mise en œuvre de l'ETP, avec l'accord préalable du patient, répond à une démarche formalisée et structurée en différentes étapes clairement définies par la HAS (Figure 1). La première étape est l'élaboration du diagnostic éducatif, lequel est aujourd'hui plus pertinemment nommé « bilan éducatif partagé » [4]. Cette étape permet d'identifier les besoins d'éducation, les attentes et les ressources du patient. L'étape suivante consiste à choisir et négocier avec le patient les priorités d'apprentissage et les compétences qu'il doit développer. Les séances seront alors planifiées et mises en œuvre selon des modalités adaptées au patient (choix des techniques et outils pédagogiques...). Enfin, une évaluation de l'impact du programme et des compétences acquises par le patient est réalisée.

\section{ETP ET DOULEUR LIEE AU CANCER}

La douleur est l'un des symptômes les plus redoutés auxquels sont confrontés les patients atteints de cancer. Sa prévalence chez les patients traités reste évaluée dans une récente métaanalyse internationale à 55\% [5]. Une enquête menée en France par l'Institut National du Cancer (INCa) rapporte que 62\% des patients en situation de cancer avancé seraient sous-traités sur le plan antalgique [6]. La douleur en oncologie reste donc insuffisamment soulagée et serait associée à une qualité de vie altérée [7]. La prise en charge des patients se complexifie dans un 
contexte où le cancer, du fait des avancées technologiques et thérapeutiques, tend à devenir une maladie chronique, traitée de plus en plus fréquemment en ambulatoire.

La prise en charge de la douleur en oncologie se heurte à différents obstacles parmi lesquels le manque de connaissance et les représentations du patient et/ou de son entourage sur la douleur et ses traitements [8]. Les obstacles se traduisent notamment par des idées reçues concernant l'utilisation des opioïdes, une sous-estimation de la douleur, une non-adhésion au traitement et une réticence à alerter les professionnels de santé si besoin [9]. Afin de les surmonter, la participation active des patients s'avère nécessaire. L'ETP visant à permettre au patient de développer les compétences pour optimiser au mieux la gestion de sa douleur, constitue ainsi une approche stratégique pertinente répondant à cette problématique. Les programmes d'éducation du patient en matière de douleur se sont développés ces dernières années, comme l'illustre la littérature. L'analyse des publications sur le sujet permet de documenter les bénéfices et d'identifier les programmes d'ETP les plus efficients [10]. En particulier, la revue systématique de Adam [11] consiste notamment en une synthèse récente de huit revues systématiques (Tableau 1, [12-19]) dont trois méta-analyses [14,16,19], répondant à une sélection méthodologique rigoureuse (PRISMA pour Preferred Reporting Items for Systematic Reviews and Meta-analyses) relatives à la recherche menée dans le domaine de la douleur du cancer pendant presque quarante années. Ce travail montre un bénéfice léger (statistiquement significatif) de l'ETP sur la connaissance de la douleur, les attitudes face à celle-ci, ainsi qu'une diminution de son intensité rapportée par le patient. S'agissant des éléments-clés entrant dans la construction d'un programme sur la douleur en oncologie, cette revue souligne l'importance de l'amélioration des connaissances sur la douleur, de la communication concernant la douleur, de son évaluation en continu, de l'amélioration de la prescription des traitements antalgiques et de l'adhésion médicamenteuse en combattant les idées reçues et de la formation aux stratégies non pharmacologiques de gestion de la douleur.

\section{DEVELOPPEMENT D'UN PROGRAMME REGIONAL D'ETP SUR LA DOULEUR LIEE AU CANCER ET DEPLOIEMENT NATIONAL}

Si les programmes d'ETP se développent actuellement en France, notamment en oncologie [20], on constate néanmoins la quasi-inexistence de recherche en matière d'ETP dans la douleur liée au cancer. Pour autant, favoriser les actions ETP dans ce domaine a été identifié comme une priorité dans le Plan Cancer III (Action 7.6) [21]. Dans cette optique, un large projet 
d'éducation thérapeutique dédié à cette thématique est actuellement en cours de développement en ex Basse-Normandie (protocole EFFADOL pour «Ensemble, Faire Face A la DOuLeur »). Sur le territoire, la prise en charge des patients atteints de cancer s'appuie notamment sur deux réseaux structurés : le Réseau régional de cancérologie (OncoBasseNormandie) et le Réseau Régional Douleur en Basse-Normandie (RRDBN) permettant ainsi la proximité et l'accessibilité pour tous. Le RRDBN s'appuie sur dix structures identifiées d'étude et de traitement de la douleur permettant une couverture géographique régionale au sein desquelles sont identifiés des binômes médecin-infirmière référents. Ces structures prennent en charge les patients douloureux chroniques selon les critères de la HAS avec une approche pluridisciplinaire et pluriprofessionnelle dans laquelle la dimension éducative n'était pas encore formalisée.

Le développement de ce projet d'ETP s'inscrit donc dans une politique régionale de santé et se structure en cinq étapes (Figure 2):

- Formation à l'ETP de professionnels de santé (10 binômes médecins/infirmières)

- Identification des attentes éducatives des patients et des proches dans le domaine de la douleur

- Conception d'un programme d'ETP sur la douleur liée au cancer

- Etude Pilote Régionale permettant d'évaluer la faisabilité, la qualité et la transférabilité du programme

- Evaluation du programme d'ETP par randomisation comparative interventionnelle au niveau national.

La première étape, initiée dès 2013 et préambule de notre démarche, a donc consisté à former un groupe régional de travail (10 binômes référents des structures identifiées douleur) à l'ETP par un organisme de formation conformément aux arrêtés régulant l'ETP (arrêté du 14 janvier 2015 modifiant l'arrêté du 02 août 2010) [22]; cette formation initiale permet une qualité minimale tout en introduisant un cadre formatif, organisé et structuré.

L'établissement du programme d'ETP sur la douleur en oncologie s'appuie sur les étapes 2 et 3 menées en parallèle, lesquelles permettent de construire un programme éducatif en adéquation avec les attentes des patients. Menée auprès de 75 patients recrutés de façon représentative de la file active des 10 structures participantes, l'étape 2 a notamment permis aux patients de hiérarchiser leurs préférences éducatives concernant les thématiques à aborder lors des ateliers. 
Ainsi, les deux items principalement retenus (à plus de 65\%) ont été «Comprendre vos douleurs » et « Mieux gérer seul vos douleurs au quotidien ». La majorité des patients (plus de $97 \%$ ) serait partante pour participer à un programme d'ETP, avec une préférence pour les séances individuelles (pour $41,2 \%$ ) ou une alternance séances collectives et individuelles $(29,4 \%)$.

Le groupe régional de travail a par conséquent élaboré un programme d'ETP répondant aux critères de qualité de la HAS et autorisé par l'Agence Régionale de Santé (ARS) en septembre 2014. Outre l'équipe éducative et un coordonnateur formé à l'ETP, des patients ressources et membres de la Ligue contre le Cancer et de l'Association Francophone pour Vaincre les Douleurs ont également été impliqués dans l'élaboration du programme tel que le préconise la HAS. Ce programme prévoit un bilan éducatif partagé et l'établissement d'un programme personnalisé d'ETP conformément aux recommandations [2]. Il comprend également trois séances éducatives proposées sous forme individuelle et/ou collective selon le souhait des patients, lesquels peuvent, s'ils le désirent, solliciter un proche pour y participer. Les compétences déclinées dans ces 3 séances sont :

- comprendre sa douleur ;

- comprendre les traitements de sa douleur et mieux gérer leurs effets indésirables ;

- s'adapter au mieux à la douleur au quotidien.

Chaque séance se compose de deux ateliers de 40 minutes environ. Le programme d'ETP repose également sur des outils pédagogiques élaborés par le groupe de travail visant à faciliter les apprentissages tels que la photo-expression, des exposés interactifs et des mises en situations sous forme de cartes interactives. Ces ressources pédagogiques communes aux 10 sites permettent, au-delà de la formation collective à l'ETP une harmonisation dans la pratique éducative. Quant au dossier éducatif intégrant les actions des soignants-éducateurs et les décisions prises avec les participants, il permet un suivi du patient et le partage d'information avec les professionnels de santé, notamment le médecin traitant.

A ce jour, plus de 30 patients ont bénéficié du programme, qu'ils ont suivi dans son intégralité. Même si l'essentiel des premiers patients a été essentiellement recruté dans les centres de référence, notamment le Centre de Lutte contre le Cancer François Baclesse (Caen), les centres de recours ont maintenant également mis en œuvre le programme d'ETP.

Enfin, l'évaluation du programme d'ETP, réalisée tant en termes de qualité et de transférabilité que d'efficacité, s'appuie sur les deux dernières étapes. Une étude pilote sera tout d'abord 
conduite au niveau régional, réalisée par les professionnels de santé du RRDBN formés préalablement à l'ETP lors de l'étape 1. La dernière étape quant à elle, permettra d'évaluer le bénéfice de l'approche éducative comparée à la prise en charge conventionnelle de la douleur grâce à une randomisation comparative interventionnelle à l'échelle nationale. Les deux étapes d'évaluation qui succèdent au programme complètent les auto-évaluations annuelles qui doivent être transmises à l'ARS. Elles permettront l'optimisation et le réajustement si besoin du programme (Figure 2) qui sera reconduit ou non après l'évaluation formalisée quadriennale de l'ARS.

\section{POINTS-CLES DES ETAPES DE RECHERCHE DU PROJET EFFADOL}

Objectifs, méthodologie et résultats attendus des différentes étapes de recherche du programme sont présentés dans le Tableau 2.

\section{POPUlation CIBLE}

Les patients susceptibles d'être inclus dans les différentes étapes du projet et de bénéficier du programme d'ETP répondent à des critères définis par le groupe de travail, en s'appuyant sur la définition d'une douleur de fond non équilibrée selon les Standards, Options et Recommandations [23]. Les patients concernés sont donc des patients présentant une douleur liée à la maladie ou à ses traitements et/ou aux séquelles de la maladie et de ses traitements (en cours ou non), traitée par antalgique depuis plus d'un mois, et d'intensité modérée à sévère, non équilibrée dans la semaine précédant leur inclusion dans le programme d'ETP. Cette douleur est caractérisée par une intensité supérieure ou égale à 4 sur une échelle de 10 ou une douleur insomniante ou plus de 4 accès douloureux paroxystiques par jour ou un retentissement sur les activités quotidiennes.

\section{REPRESENTATIVITE DES DIVERS BASSINS DE POPULATION}

Un des points importants de ce projet est la philosophie affichée par le RRDBN de permettre grâce au réseau la proximité et l'accessibilité pour tous au programme. L'objectif à cet égard est donc d'harmoniser les pratiques et de permettre en matière d'ETP des soins de proximité identiques et efficients, quel que soit le lieu de résidence du patient, non limités au seul cadre 
des grands hôpitaux. Le nombre de patients nécessaires pour répondre aux questions de recherche des étapes régionales (2 et 4) est donc également réparti entre les structures universitaires et les établissements périphériques de façon à caractériser au mieux les caractéristiques éducatives dans les divers bassins de population de la région.

\section{Evaluation DE LA DOULEUR ET DE SON RETENTISSEMENT - CHOIX DU CRITERE DE JUGEMENT}

Le critère de jugement généralement choisi dans les études pour évaluer l'efficacité de la démarche d'ETP est la diminution de l'intensité de la douleur (mesurée par auto-évaluation à l'aide des échelles). Pour autant, dans un contexte d'éducation sur la douleur, l'évaluation du retentissement de celle-ci sur les activités quotidiennes semble un paramètre moins fluctuant que l'intensité douloureuse, plus réaliste et aussi plus pertinent en terme d'objectif. A cet égard, le Questionnaire Concis sur les Douleurs (QCD), traduction française du Brief Pain Inventory (BPI, [24]) est très couramment utilisé dans les protocoles de recherche [10]. Ce questionnaire, notamment sous sa version courte, présente l'avantage d'être validé en français [25] et la HAS préconise l'utilisation de la sous-échelle 9 pour évaluer la qualité de vie des patients douloureux [26].

Ce questionnaire, outre l'évaluation de l'intensité de la douleur, sa localisation et son soulagement, permet en effet une auto-évaluation rapide du retentissement de la douleur sur les activités quotidiennes, en considérant deux sous-dimensions (affect et activité) à l'aide de sept items (Figure 3). Un score est établi en faisant la moyenne des réponses à cette sous-échelle. Le bénéfice de l'ETP sur le retentissement de la douleur est défini par une diminution de 2 points de ce score (sur une échelle de 10) entre les valeurs mesurées avant et à la fin du programme d'éducation, correspondant, selon les données de la littérature, à une amélioration cliniquement significative [27]. Le QCD permet aussi de calculer le « Pain Management Index », indice de gestion de la douleur qui, en combinant l'intensité de la douleur et le traitement antalgique, constitue une évaluation de l'utilisation adéquate des antalgiques [28]. Parmi les autres critères, sont intégrés le retentissement émotionnel grâce à l'échelle validée HAD (Hospital Anxiety and Depression Scale, [29]) ainsi que l'évaluation qualitative de la douleur par le questionnaire de Saint-Antoine validé lui aussi sous sa forme abrégée [30], y compris en français [31]. 


\section{OUTILS CHOISIS POUR EVALUER LA DIMENSION EDUCATIVE DU PROGRAMME}

Un des critères les plus évalués dans les études publiées et montrant un bénéfice de l'ETP est l'acquisition par le patient de connaissances et de compétences concernant sa douleur et son traitement [10,11]. Les étapes de recherche utilisent également un questionnaire de connaissances du patient sur la douleur et son traitement, construit par le groupe de travail et proposé au début et en toute fin du programme d'ETP.

Des questionnaires non validés mais répondant spécifiquement à des critères éducatifs évaluent l'auto-efficacité du patient à participer activement à la gestion de sa douleur et à communiquer de manière optimale avec les soignants [32]. Y sont associées des échelles de perception par le patient d'un changement par rapport à sa santé [33], ici plus particulièrement adaptées à sa douleur et à son retentissement suite au programme d'ETP, échelles pertinentes et sensibles utilisées de manière rétrospective.

Les proches qui auront répondu à la sollicitation des patients verront leur qualité de vie évaluée par un auto-questionnaire spécifiquement développé en oncologie et validé en français (CarGoQoL, [34]).

EVALUATION DE LA SATISFACTION DES PARTICIPANTS ET EDUCATEURS SUR LE PROGRAMME

De plus, il est important d'évaluer la satisfaction des patients à l'égard de la prise en charge éducative de leur douleur [35] et de la qualité du programme car ces critères permettront l'optimisation de ce dernier. Afin de limiter la surcharge de travail des soignants-éducateurs induite par la démarche de recherche et de ne pas sur-solliciter les patients en multipliant les questionnaires, des outils communs sont utilisés pour évaluer la satisfaction des participants au programme dans le cadre de la recherche et des évaluations préconisées par la HAS. Ces outils ont été adaptés à la douleur cancéreuse à partir de ceux proposés par l'Espace Régional d'Education Thérapeutique (ERET,[36]), structure régionale ressource dont la mission est notamment d'harmoniser la pratique de l'ETP et son évaluation sur le territoire. Les questionnaires portent sur les modalités des ateliers (durée, rythme...), les bénéfices apportés concernant la gestion de la douleur, la qualité des éducateurs et la clarté de leurs messages... 
L'évaluation du programme et de ses bénéfices sur les patients est également menée auprès des soignants-éducateurs. On évalue également les conséquences du programme sur l'équipe éducative en termes de fonctionnement, de modification des pratiques, d'intégration au sein de l'offre locale de soins, concernant notamment la communication avec le médecin traitant.

\section{CHOIX DU DESIGN STEP-WEDGE}

L'impact du programme et le bénéfice de l'approche éducative sont évalués au décours d'une étude clinique randomisée en comparant l'approche éducative et la prise en charge «conventionnelle» de la douleur (étape5). Dans les études cliniques interventionnelles, les essais randomisés contrôlés sont considérées comme le «gold standard » de l'évaluation et à ce titre sont principalement utilisés pour valider l'impact de l'approche éducative. Dans ce projet, un design randomisé particulier appelé step-wedge [37-39] a été choisi pour éviter le risque de contamination entre les deux groupes et réduire le risque de biais. En effet, il n'est pas envisageable de mettre en place simultanément par des mêmes soignants deux modes de prise en charge différents (ETP et prise en charge conventionnelle) étant donné qu'il s'agit ici d'évaluer une pratique impliquant une modification de posture du soignant. Ce design permet d'échelonner, à l'échelle des structures, la formation des soignants et par conséquent celle de l'intervention éducative selon les centres d'inclusion, la randomisation s'effectuant alors selon les centres (par grappe ou « cluster ») et non plus selon les patients. Au début de l'étude, il est prévu d'inclure les patients du territoire bas-normand, cette première phase s'adressant exclusivement à des patients bénéficiant de l'approche éducative. Puis, les 5 autres structures participantes au projet hors de la région sont inclues progressivement pour mettre en place le step-wedge ce qui permettra de comparer l'approche conventionnelle (avant la formation) à l'approche éducative (Figure 4). La formation est assurée par l'IPCEM, organisme de référence en la matière [40] et financée dans le cadre du projet, ce qui garantit l'homogénéité des formations reçues dans tous les centres participants. Elle est complétée par une formation spécifique par le groupe de travail au programme d'ETP de la douleur en oncologie.

\section{CONCLUSION}

L'originalité et la force de ce programme reposent sur un travail collaboratif entre des acteurs de santé et une démarche de recherche s'appuyant sur des méthodologies robustes visant à objectiver les bénéfices apportés au patient. 
En effet, au terme de ces cinq étapes identifiées, l'objectif est de démontrer l'efficacité du programme d'ETP à améliorer les connaissances et compétences des patients dans le domaine de la douleur liée au cancer et par conséquent leur permettre de mieux gérer leur douleur au quotidien. Grâce à l'analyse et à la caractérisation des résultats obtenus, le programme pourra également être optimisé et les procédures suivies adaptées ou corrigées si nécessaire. La propagation de l'approche éducative dans les territoires de santé au sein des structures hospitalières régionales ou nationales, permettra son accès à tout patient atteint de cancer, dans un contexte de proximité favorisé par les réseaux, au-delà du seul cadre des grands hôpitaux.

A l'issue du projet, les connaissances en matière de résultats de l'ETP sur la problématique étudiée seront enrichies et en ce sens le projet contribue plus largement à la diffusion de la démarche éducative. Celle-ci s'accompagne d'une modification des pratiques apportant un bénéfice réciproque pour les patients et pour les soignants-éducateurs. Il y a redéfinition des rôles de part et d'autre avec engagement dans des décisions de soins collaboratives, sollicitant la participation active des patients.

La douleur du cancer est un challenge national et mieux la gérer un enjeu de santé publique. A cet égard, l'ETP est un atout pour permettre de mieux évaluer, traiter et comprendre la problématique. C'est un véritable levier pour la transformation culturelle à laquelle doivent prendre part ensemble agences gouvernementales, financeurs, professionnels de santé formés à l'ETP et patients. C'est aussi une stratégie à l'échelle des populations et un enjeu dans la qualité des soins. En termes de santé publique en effet, la mise en place des programmes d'ETP a pour objectif une rationalisation du recours aux soins avec diminution des coûts de santé et des recours aux hospitalisations. Les aspects médico-économiques sont des pistes complémentaires à explorer.

\section{REMERCIEMENTS}

Les auteurs remercient le Pr Khaled Meflah (Directeur du Centre F. Baclesse), l'Espace Régional d'Education Thérapeutique (ERET) Basse-Normandie et le Dr Loudiyi-Mehdaoui (ARS) pour leur soutien. Les auteurs remercient pour leur soutien financier la Fondation Apicil et l'Association ARDCOM (étape 1), la société TAKEDA (étape 2), le Comité départemental de la Ligue contre le Cancer de Basse-Normandie (étape 4) et l'INCa (Appel d'offre RISP15- 
007_FP, étape 5). Nous remercions aussi les patients et représentants d'associations qui ont contribué à la construction de ce projet et tout particulièrement Jocelyne Padéri (Association Francophone pour Vaincre les Douleurs) pour son implication attentive et bienveillante. L'ensemble des patients et des membres de leur entourage qui ont accepté (étape 2) et accepteront (étapes 4 et 5) de participer aux essais cliniques mis en œuvre dans le cadre de ce large programme de recherche est également remercié.

Conflits d'intérêts : aucun 


\section{REFERENCES}

[1] OMS. Rapport de l'OMS-Europe, publié en 1996, Therapeutic Patient Education Continuing Education Programmes for Health Care Providers in the field of Chronic Disease, traduit en français en 1998.

http://www.euro.who.int/_data/assets/pdf_file/0007/145294/E63674.pdf. Accès au site le $20 / 12 / 2017$.

[2] HAS. Education thérapeutique du patient - définition, finalités et organisation, juin 2007- https://www.has-sante.fr/portail/upload/docs/application/pdf/etp__definition_finalites_-_recommandations_juin_2007.pdf. Accès au site le 20/12/2017.

[3] Loi HPST. Loi n ${ }^{\circ} 2009-879$ du 21 juillet 2009https://www.legifrance.gouv.fr/affichTexte.do?cidTexte=JORFTEXT000020879475\&c ategorieLien=id. Accès au site le 20/12/2017.

[4] Sandrin-Berthon B. Diagnostic éducatif ou bilan éducatif partagé ? Med Mal Metab 2010;4:38-43.

[5] Van den Beuken-van Everdingen MHJ, Hochstenbach LMJ, Joosten EAJ, TjanHeijnen VCG, Janssen DJA. Update on Prevalence of Pain in Patients With Cancer: Systematic Review and Meta-Analysis. J Pain Symptom Manage 2016;51:1070-90.

[6] Site de l'Institut National du Cancer (INCA), mars 2012, http://www.ecancer.fr/publications/73-soins/390-enquete-nationale-2010-sur-la-prise-en-charge-dela-douleur-chez-des-patients-adultes-atteints-de-cancer-synthese.Accès au site le 20/12/2017.

[7] Kroenke K, Theobald D, Wu J, Loza JK, Carpenter JS, et al. The association of depression and pain with health-related quality of life, disability, and health care use in cancer patients. J Pain Symptom Manage 2010;40:327-41.

[8] Brant JM. The global experience of cancer pain. Asian Pac J Cancer Prev 2010;11 Suppl $1: 7-12$.

[9] Kwon JH. Overcoming barriers in cancer pain management. J Clin Oncol 2014;32:172733.

[10] Prevost V, Delorme C, Grach MC, Chvetzoff G, Hureau M. Therapeutic Education in Improving Cancer Pain Management: A Synthesis of Available Studies. Am J Hosp Palliat Care 2016;33:599-612.

[11] Adam R, Bond C, Murchie P. Educational interventions for cancer pain. A systematic review of systematic reviews with nested narrative review of randomized controlled 
trials. Patient Educ Couns 2015;98:269-82.

[12] Allard P, Maunsell E, Labbé J, Dorval M. Educational interventions to improve cancer pain control: a systematic review. J Palliat Med 2001;4:191-203.

[13] Goldberg GR, Morrison RS. Pain management in hospitalized cancer patients: a systematic review. J Clin Oncol 2007;25:1792-801.

[14] Bennett MI, Bagnall AM, José Closs S. How effective are patient-based educational interventions in the management of cancer pain? Pain 2009;143:192-9.

[15] Oldenmenger WH, Sillevis Smitt PA, van Dooren S, Stoter G, van der Rijt CC. A systematic review on barriers hindering adequate cancer pain management and interventions to reduce them: a critical appraisal. Eur J Cancer 2009;45:1370-80.

[16] Cummings GG, Olivo SA, Biondo PD, Stiles CR, Yurtseven O, Fainsinger RL et al. Effectiveness of knowledge translation interventions to improve cancer pain management. J Pain Symptom Manage 2011;41:915-39.

[17] Koller A, Miaskowski C, De Geest S, Opitz O, Spichiger E. A systematic evaluation of interventions of content, structure, and efficacy to improve patients' self-management of cancer pain. J Pain Symptom Manage 2012;44:264-84.

[18] Ling CC, Lui LY, So WK. Do educational interventions improve cancer patients' quality of life and reduce pain intensity? Quantitative systematic review. J Adv Nurs 2012,68:511-20.

[19] Jho, H. J., Myung, S.-K., Chang, Y.-J., Kim, D.-H., Ko, D. H. Efficacy of pain education in cancer patients: a meta-analysis of randomized controlled trials. Supportive care in cancer: official journal of the Multinational Association of Supportive Care in Cancer 2013;21:1963-71.

[20] http://www.educationtherapeutique-idf.org/_front/Pages/page.php. Accès au site le $20 / 12 / 2017$.

[21] http://www.e-cancer.fr/Plan-cancer/Plan-cancer-2014-2019-priorites-et-objectifs. Accès au site le 20/12/2017.

[22] https://www.legifrance.gouv.fr/eli/arrete/2015/1/14/AFSP1501146A/jo/texte. Accès au site le 20/12/2017.

[23] Poulain P, Michenot N, Ammar D, Delorme C, Delorme T, Diquet B et al. Mise au point sur l'utilisation du fentanyl transmuqueux chez le patient présentant des douleurs d'origine cancéreuse (version longue). Doul. et Analg 2012;25:102-17.

[24] Cleeland CS \& Ryan KM. Pain assessment : global use of the Brief Pain Inventory, Ann Acad Med Singapore 1994;13:129-38. 
[25] Larue F, Colleau SM, Brasseur L, Cleeland CS. Multicentre study of cancer pain and its treatment in France. BMJ 1995;310:1034-7.

[26] ANAES, Evaluation et suivi de la douleur chronique chez l'adulte en médecine ambulatoire, Service des Recommandations et Références professionnelles, février 1999.

[27] Cleeland CS, Body JJ, Stopeck A, von Moos R, Fallowfield L, Mathias SD et al. Pain outcomes in patients with advanced breast cancer and bone metastases: results from a randomized, double-blind study of denosumab and zoledronic acid. Cancer 2013;119:832-8.

[28] Zelman DC, Cleeland CS, Howland EW. Factors in appropriate pharmacological management of cancer pain: a cross-institutional investigation. Pain 1987(Suppl): S136.

[29] Guelfi JD. L'évaluation clinique standardisée en psychiatrie. Tome 1. Psychopathologie générale, dépression, anxiété et anxiodépression. Boulogne: Éditions Médicales Pierre Fabre; 1996

[30] Boureau F, Luu M, Doubrère JF. Comparative study of the validity of four French McGill Pain Questionnaire (MPQ) versions. Pain 1992;50:59-65.

[31] Marquié L, Duarte L-R, Mauriès V, Izard P, Pouymayou J. Les caractéristiques psychométriques du Questionnaire de douleur de Saint-Antoine en consultation d'algologie chez les personnes atteintes de cancer. Doul et Analg 2008;21:52-61.

[32] Street RL Jr, Slee C, Kalauokalani DK, Dean DE, Tancredi DJ, Kravitz RL. Improving physician-patient communication about cancer pain with a tailored education-coaching intervention. Patient Educ Couns 2010;80:42-7.

[33] Fischer D, Stewart A L, Bloch DA, Lorig K, Laurent D, Holman H. Capturing the patient's view of change as a clinical outcome measure. JAMA: the journal of the American Medical Association 1999;282:1157-62.

[34] Minaya P, Baumstarck K, Berbis J, Goncalves A, Barlesi F, Michel G et al. The CareGiver Oncology Quality of Life questionnaire (CarGOQoL): development and validation of an instrument to measure the quality of life of the caregivers of patients with cancer. Eur J Cancer 2012;48:904-11.

[35] Barlési F, Duffaud F, Doddoli C, Gimenez C, Auquier P, Favre R et al. [Impact of a brochure on pain destined for thoracic oncology patients]. Presse Med. 2004;33(19 Pt 1):1313-8.

[36] http://www.eretbn.org/\#!productions/cu231. Accès au site le 20/12/2017 
[37] Rasmussen CD, Holtermann A, Mortensen OS, Søgaard K, Jørgensen MB. Prevention of low back pain and its consequences among nurses' aides in elderly care: a steppedwedge multi-faceted cluster-randomized controlled trial. BMC Public Health 2013;13:1088.

[38] Hussey MA, Hughes JP. Cluster randomized crossover trials: design and analysis of the stepped wedge design. Contemp Clin Trials 2007;28:182-91.

[39] Mdege ND, Man MS, Taylor CA, Torgerson DJ. Systematic review of stepped wedge cluster randomized trials shows that design is particularly used to evaluate interventions during routine implementation.J Clin Epidemiol 2011;64:936-48.

[40] http://www.ipcem.org/. Accès au site le 20/12/2017. 
Tableau 1 : Synthèse des effets des programmes éducatifs mesurés chez les patients dans les revues systématiques analysées d'après Adam et al.

\begin{tabular}{|c|c|c|}
\hline $\begin{array}{l}\text { AUTEURS REFERENE, ANNEE (NOMBRE } \\
\text { D'ETUDES INCLUS DANS LA REVUE) }\end{array}$ & CRITERES d'EVALUATION PRINCIPAUX & RESULTATS PRINCIPAUX APPORTES PAR L'ETP \\
\hline $\begin{array}{l}\text { Allard et al. }{ }^{[12]}, 2001 \\
\text { (33) }\end{array}$ & $\begin{array}{l}\text { Intensité de la douleur, connaissances et } \\
\text { comportements sur la douleur des } \\
\text { professionnels et des patients, gestion et } \\
\text { soulagement de la douleur, qualité de vie }\end{array}$ & $\begin{array}{l}\text { Amélioration des connaissances et comportements des professionnels de santé face à la douleur } \\
\text { sans impact sur l'intensité de la douleur des patients; efficacité des interventions infirmières pour } \\
\text { améliorer les connaissances et compétences des patients, amélioration du soulagement de la } \\
\text { douleur dans les } 5 \text { études où il a été évalué, intérêt d'utiliser un agenda journalier de la douleur } \\
\text { pour permettre le réajustement du traitement. }\end{array}$ \\
\hline $\begin{array}{l}\text { Goldberg \& Morrison }{ }^{[13]}, 2007 \\
\text { (5) }\end{array}$ & $\begin{array}{l}\text { Intensité de la douleur, connaissances des } \\
\text { infirmières et comportement, satisfaction du } \\
\text { patient }\end{array}$ & $\begin{array}{l}\text { Amélioration des connaissances et des comportements des infirmières face à la douleur; douleur } \\
\text { mieux renseignée dans les dossiers médicaux; amélioration de la satisfaction des patients; pas } \\
\text { d'amélioration systématique de l'intensité de la douleur des patients. }\end{array}$ \\
\hline $\begin{array}{l}\text { Bennett et al. }[14], 2009 \\
\text { (21) }\end{array}$ & $\begin{array}{l}\text { Intensité de la douleur et retentissement sur le } \\
\text { quotidien, connaissances et comportement du } \\
\text { patient, adhésion au traitement et autonomie du } \\
\text { patient, qualité de vie }\end{array}$ & $\begin{array}{l}\text { Amélioration des connaissances et comportements du patient face à la douleur; diminution de } \\
\text { l'intensité de la douleur mais pas d'impact sur l'adhésion médicamenteuse ni sur le retentissement } \\
\text { de la douleur sur les activités quotidiennes. }\end{array}$ \\
\hline $\begin{array}{l}\text { Oldenmenger et al.[15], } 2009 \\
\text { (16) }\end{array}$ & $\begin{array}{l}\text { Intensité de la douleur, connaissances des } \\
\text { patients et des professionnels, barrières, }\end{array}$ & $\begin{array}{l}\text { Barrières les plus fréquemment évoquées par les patients et par les professionnels de santé : } \\
\text { manque de connaissances, évaluation inadéquate de la douleur, idées fausses concernant la } \\
\text { douleur; diminution de l'intensité de la douleur des patients dans } 7 \text { essais randomisés sur } 11 .\end{array}$ \\
\hline
\end{tabular}




\begin{tabular}{|c|c|c|}
\hline & $\begin{array}{l}\text { adhésion aux analgésiques, gestion de la } \\
\text { douleur. }\end{array}$ & \\
\hline $\begin{array}{l}\text { Cummings et al. }{ }^{[16]}, 2011 \\
\text { (26) }\end{array}$ & $\begin{array}{l}\text { Changement de comportement (professionnels } \\
\text { de santé), intensité et impact de la douleur, } \\
\text { qualité de vie, satisfaction avec le traitement, } \\
\text { changement de traitement antalgique. }\end{array}$ & $\begin{array}{l}\text { Grâce à la transmission de connaissances au personnel soignant, aux patients et aux professionnels } \\
\text { de santé, amélioration (non systématique) des connaissances, compétences et comportements en } \\
\text { ce qui concerne le contrôle de la douleur et ses conséquences. }\end{array}$ \\
\hline $\begin{array}{l}\text { Koller et al. }{ }^{[17]}, 2012 \\
\text { (24) }\end{array}$ & $\begin{array}{l}\text { Analyse qualitative pour définir la structure et le } \\
\text { contenu des interventions d'éducation } \\
\text { thérapeutique }\end{array}$ & $\begin{array}{l}\text { Réductions significatives et cliniques de l'intensité de la douleur dans } 9 \text { études sur 24; pas } \\
\text { d'association particulière entre ces résultats et des éléments spécifiques de l'éducation. }\end{array}$ \\
\hline $\begin{array}{l}\text { Ling et al. }{ }^{[18]}, 2012 \\
\text { (4) }\end{array}$ & $\begin{array}{l}\text { Intensité de la douleur, qualité de vie, } \\
\text { interférence de la douleur sur la vie quotidienne }\end{array}$ & $\begin{array}{l}\text { Diminution de l'intensité de la douleur dans } 2 \text { études sur } 4 \text {; pas d'amélioration significative de son } \\
\text { retentissement sur les activités quotidiennes ni de la qualité de vie dans aucune des études. }\end{array}$ \\
\hline $\begin{array}{l}\text { Jho et al. }{ }^{[19]}, 2013 \\
\text { (12) }\end{array}$ & Intensité de la douleur & Légère diminution significative de l'intensité de la douleur. \\
\hline
\end{tabular}


Tableau 2 : Objectifs, méthodologie et résultats attendus dans les étapes de recherche du projet EFFADOL

\begin{tabular}{|c|c|c|c|}
\hline & ObJectifs & METHODOLOGIE & RESULTATS ATTENDUS \\
\hline Etape 2 & $\begin{array}{l}\text { Identifier les attentes } \\
\text { éducatives et les besoins des } \\
\text { patients et de leurs proches } \\
\text { sur la douleur liée au cancer }\end{array}$ & $\begin{array}{l}\text { Recueil de questionnaires construits par le groupe de travail concernant la } \\
\text { hiérarchisation des besoins et les modalités et l'organisation des séances... } \\
\text { Choix de critères d'inclusion des patients. } \\
\text { Evaluation de la douleur et de son retentissement par le QCD. } \\
\text { Nombre de sujets nécessaire : } 75 \text { patients (chargés de solliciter leur proche), répartis } \\
\text { selon l'importance relative des } 10 \text { structures régionales impliquées. }\end{array}$ & $\begin{array}{l}\text { Construction d'un programme adapté aux attentes } \\
\text { identifiées. } \\
\text { Optimisation des critères de sélection des patients à } \\
\text { inclure dans le programme et évaluation de la } \\
\text { pertinence du QCD pour évaluer la douleur. }\end{array}$ \\
\hline Etape 4 & $\begin{array}{l}\text { Evaluer la faisabilité, la } \\
\text { qualité et la transférabilité } \\
\text { du programme }\end{array}$ & $\begin{array}{l}\text { Etude interventionnelle prospective multicentrique régionale. } \\
\text { Critères de jugement : proportion de patients adhérents au programme personnalisé, } \\
\text { homogénéité de l'adhésion selon les bassins de population. } \\
\text { Satisfaction des patients, des proches, des soignants-éducateurs et concordance de } \\
\text { leurs attentes. } \\
\text { Compétences d'auto-soins et d'adaptation acquises par le patient sur sa douleur. } \\
\text { Douleur et de son retentissement. } \\
\text { Validité des objectifs et techniques pédagogiques. }\end{array}$ & $\begin{array}{l}\text { Optimisation du programme. } \\
\text { Validation de sa transférabilité avant l'étude de } \\
\text { réplication à l'échelle nationale. } \\
\text { Validation de l'adhésion des patients au programme en } \\
\text { tenant compte des divers bassins de population de la } \\
\text { région. } \\
\text { Validation et optimisation des outils et critères à } \\
\text { mettre en œuvre dans l'étape } 5\end{array}$ \\
\hline
\end{tabular}




\begin{tabular}{|c|c|c|c|}
\hline & & $\begin{array}{l}\text { Nombre de sujets nécessaire : } 90 \text { patients répartis selon l'importance relative des } 10 \\
\text { structures régionales impliquées }\end{array}$ & \\
\hline Etape 5 & $\begin{array}{l}\text { Réplication et évaluation des } \\
\text { bénéfices du programme }\end{array}$ & $\begin{array}{l}\text { Randomisation comparative interventionnelle (step-wedge). } \\
\text { Critère de jugement : diminution du retentissement de la douleur sur les activités } \\
\text { quotidiennes (QCD). } \\
\text { Autres items du QCD; qualité de vie. } \\
\text { Satisfaction du patient sur le programme et ses effets et sa perception d'un } \\
\text { changement dans sa santé. } \\
\text { Acquisition de compétences par le patient concernant notamment son auto- } \\
\text { efficacité, sa participation active à la gestion de sa douleur. } \\
\text { Nombre de sujets nécessaires : } 200 \text { patients issus de } 6 \text { structures de recrutement } \\
\text { (dont le RRDBN). }\end{array}$ & $\begin{array}{l}\text { Démonstration de l'efficacité de l'ETP (versus prise en } \\
\text { charge conventionnelle de la douleur) pour permettre } \\
\text { au patient de mieux gérer sa douleur. } \\
\text { Diffusion de la démarche éducative avec modification } \\
\text { des pratiques dans un contexte d'accessibilité aux } \\
\text { soins. }\end{array}$ \\
\hline
\end{tabular}

QCD : Questionnaire Concis sur les Douleurs ; RRDBN : Réseau Régional Douleur en Basse-Normandie 
Figure 1 : Mise en œuvre de l'éducation thérapeutique du patient structurée selon la Haute Autorité de Santé [2]

\begin{tabular}{|l|l|l|l|l|}
\hline $\begin{array}{l}\text { Élaborer un } \\
\text { diagnostic } \\
\text { éducatif }\end{array}$ & $\begin{array}{l}\text { Définir un programme } \\
\text { personnalisé d'ETP } \\
\text { avec priorités } \\
\text { d'apprentissage }\end{array}$ & $\begin{array}{l}\text { Planifier et mettre en } \\
\text { œuvre les séances } \\
\text { d'ETP individuelle, } \\
\text { collective, ou en } \\
\text { alternance }\end{array}$
\end{tabular}$\longrightarrow$\begin{tabular}{l}
$\begin{array}{l}\text { Réaliser une évaluation } \\
\text { des compétences } \\
\text { acquises, du déroulement } \\
\text { du programme }\end{array}$ \\
\hline
\end{tabular}


Figure 2 : Conception et stratégie du projet EFFADOL à cinq étapes

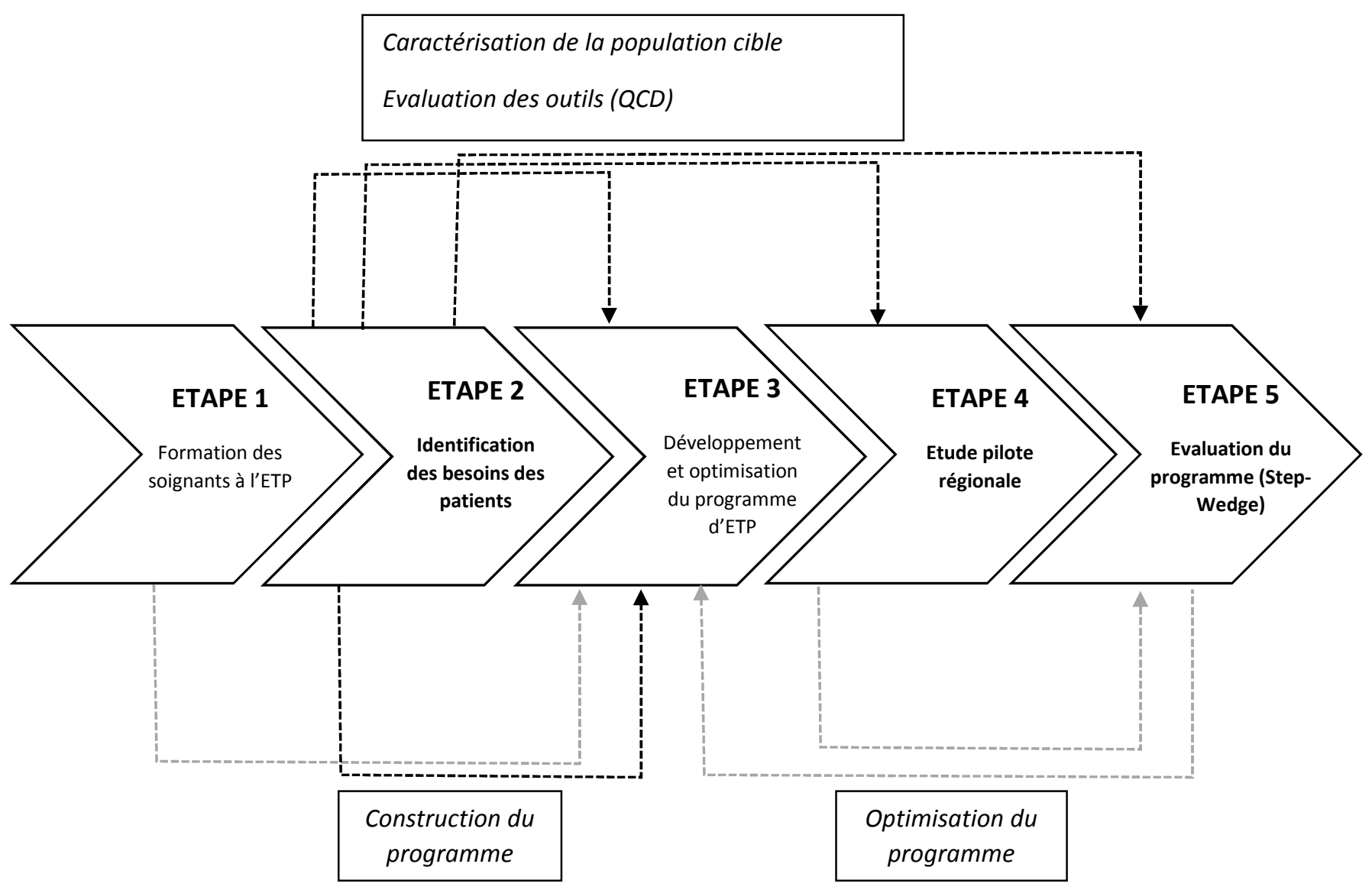

ETP: Education Thérapeutique du Patient; QCD: Questionnaire Concis sur la Douleur 
Figure 3 : Sous-échelle 9 du Questionnaire Concis sur la Douleur [25]

9. Entourez le chiffre qui décrit le mieux comment, la semaine dernière, la douleur a gêné votre :

A) Activité générale

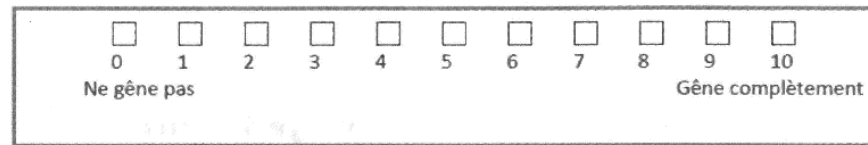

B) Humeur

\begin{tabular}{|ccccccccccc|}
\hline & $\square$ & $\square$ & $\square$ & $\square$ & $\square$ & $\square$ & $\square$ & $\square$ & $\square$ \\
0 & 1 \\
Ne gêne pas & 2 & 3 & 4 & 5 & 6 & 7 & 8 & $\begin{array}{c}\square \\
10 \\
\text { Gêne complètement }\end{array}$ \\
\hline
\end{tabular}

C) Capacité à marcher

$\begin{array}{|ccccccccccc|}\square & \square & \square & \square & \square & \square & \square & \square & \square & \square & \square \\ 0 & 1 & 2 & 3 & 4 & 5 & 6 & 7 & 8 & 9 & 10 \\ \text { Ne gêne pas } & & & & & & & & \text { Gêne complètement }\end{array}$

D) Travail habituel (y compris à l'extérieur de la maison et les travaux domestiques)

\begin{tabular}{|ccccccccccc|}
\hline & $\square$ & $\square$ & $\square$ & $\square$ & $\square$ & $\square$ & $\square$ & $\square$ & $\square$ & $\square$ \\
0 & 1 & 2 & 3 & 4 & 5 & 6 & 7 & 8 & 9 & 10 \\
Ne gêne pas & & & & & & & & Gêne complètement
\end{tabular}

E) Relations avec les autres

\begin{tabular}{|ccccccccccc|}
\hline$\square$ & $\square$ & $\square$ & $\square$ & $\square$ & $\square$ & $\square$ & $\square$ & $\square$ & $\square$ & $\square$ \\
0 & 1 & 2 & 3 & 4 & 5 & 6 & 7 & 8 & 9 & 10 \\
Ne gêne pas & & & & & & & & Gêne complètement \\
\hline
\end{tabular}

F) Sommeil

\begin{tabular}{|cccccccccccc|}
\hline$\square$ & $\square$ & $\square$ & $\square$ & $\square$ & $\square$ & $\square$ & $\square$ & $\square$ & $\square$ & $\square$ \\
0 & 1 & 2 & 3 & 4 & 5 & 6 & 7 & 8 & 9 & 10 \\
Ne gêne pas & & & & & & & & Gêne complètement
\end{tabular}

G) Goût de vivre

\begin{tabular}{|cccccccccccc}
$\square$ & $\square$ & $\square$ & $\square$ & $\square$ & $\square$ & $\square$ & $\square$ & $\square$ & $\square$ & $\square$ \\
0 & 1 & 2 & 3 & 4 & 5 & 6 & 7 & 8 & 9 & 10 \\
Ne gêne pas & & & & & & & & Gêne complètement
\end{tabular}


Figure 4 : Représentation schématique du design step-wedge

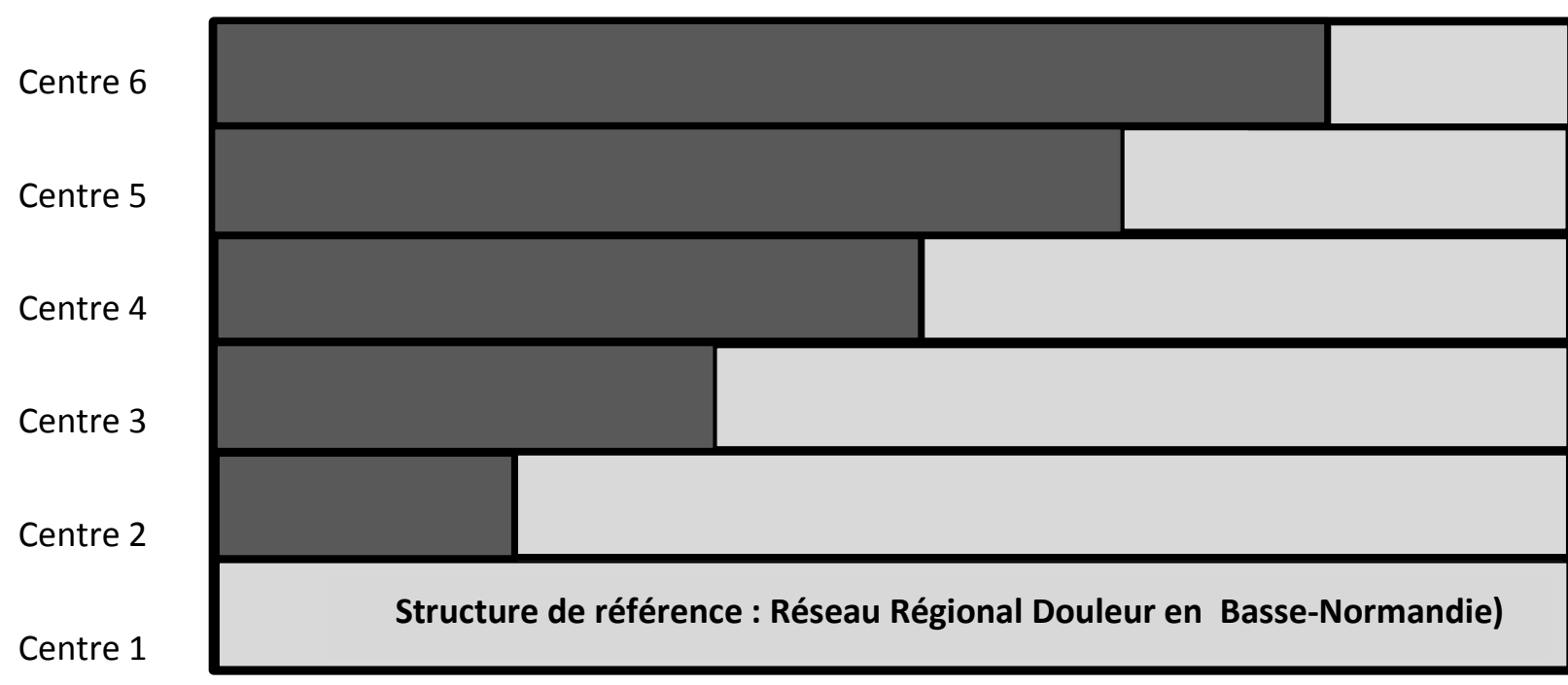

Temps (mois)

Prise en charge conventionnelle

Intervention ETP de la douleur 